\title{
Re-Sterilisation of Single-Use Telemetric Devices
}

\author{
Leonie Zieglowski Anna M. Kümmecke René H. Tolba Lisa Ernst \\ Institute for Laboratory Animal Science and Experimental Surgery, RWTH Aachen University, Faculty of Medicine, \\ Aachen, Germany
}

\section{Keywords}

Hydrogen peroxide - Gas re-sterilisation - Telemetric

devices $\cdot$ Implants $\cdot$ Single-use

\begin{abstract}
Implantable telemetric transponders for contactless measurement of physiological parameters are often used in animal-based research. After explantation, single-use devices cannot be re-implanted because of non-validated functionality and necessary re-sterilisation. This is disadvantageous because the battery life would enable a second implantation cycle in another animal. To save costs and time taken for the manufacturer's refurbishing process, we validated and implemented a re-sterilisation protocol for single-use transponders using hydrogen peroxide gas. The described protocol was established with models, i.e., for large $(n=7)$ and small $(n=3)$ animals, of telemetric device from 2 different manufacturers (Data Science International and EMKA). All transponders, prepared according to the protocol, were previously implanted subcutaneously in the flank of pigs or rats for a duration of 21 days. Our investigations demonstrate that disinfection only is not sufficient against bacterial contamination and that sterility can only be achieved by addi-
\end{abstract}

tional gas sterilisation with hydrogen peroxide. Furthermore, re-implantation of the re-sterilised transponders into pigs caused neither undesired tissue reactions along the transponder nor impairment of the measured values when compared to the first implantation and after necropsy in 4 cases. We were able to demonstrate that, using our protocol, re-implantation of reprocessed single-use telemetric devices can be performed without compromising transponder quality.

(c) 2021 S. Karger AG, Basel

\section{Introduction}

Telemetric transponder devices are designed to monitor and collect physiological data from conscious and freely moving animals of various species $[1,2]$. Surgically implantable devices are able to measure vital parameters immediately and continuously within different fields of animal science such as cardiovascular, respiratory, or surgical studies [3]. Data is acquired and trans-

R.H.T. and L.E. contributed equally to this work.

\section{Karger $\stackrel{2}{=}$}

Correspondence to:

René H. Tolba, rtolba@ukaachen.de 


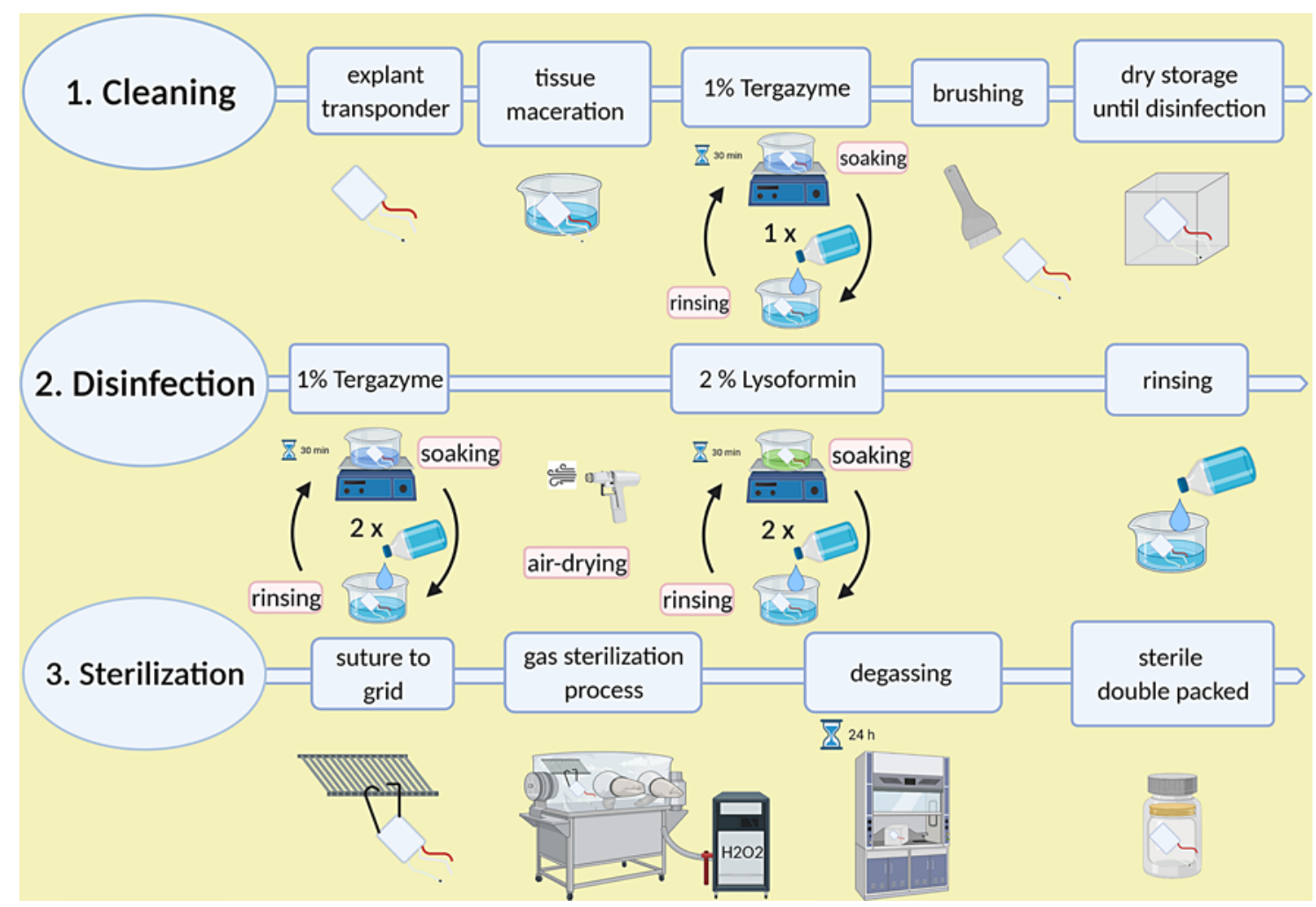

Fig. 1. Step-by-step illustration of the 3 phases of sterilization: cleaning, disinfection, and sterilisation (created with BioRender.com).

mitted wirelessly to receivers and converted, and the recorded telemetric signals can be analysed using manufacturer-specific software $[4,5]$. The life span of a transponder device is primarily determined by its battery capacity. Consecutively, this is affected by the size of the transponder, adapted to the respective animal species, as well as by the number of measuring channels. For both small and large animals, the battery life is often limited to 4-48 weeks, also depending on the days of continuous recording, the data rate set, and the sampling frequency. Individual battery life spans are listed on the homepages of the customers and the respective data sheets of the devices.

The short usage time implies that these devices are mostly used for short-term studies and are therefore recommended by the manufacturer for single use only.

Although in most cases battery power is still available after single use, repeated implantation is not recommended without prior expensive refurbishing by the manufacturer. However, repeated usage with sufficient battery capacity would be desirable from an economic and environmental viewpoint. Refurbishment includes inspecting technical integrity, cleaning, and sterilisation. Therefore, we developed a gas sterilisation protocol that can be easily implemented.

Unfortunately, most manufacturers do not offer their own refurbishment service, and cost and transport requirements enormously increase the effort. This usually means discarding the device and investing in a new one. If the refurbishment is to be performed by the customer, the components of the transponder, with different surfaces and materials (biocompatible silicone and medicalgrade polymers), pose certain challenges. Simple superficial disinfection is not sufficient to remove biofilm and bacterial contamination from the entire surface. Moreover, it is not sufficient to remove persistent bacteria; this could lead to resistant bacteria and reinfection in the next animal. Here, it is irrelevant whether the contaminating pathogens originate from animal husbandry and contact surfaces or are transferred from previous implantations [6-8]. To prevent potential infections, wound-healing disorders, and measurement impairments, it is therefore important to exclude biofilms and germ transmission in this set-up. 


\begin{tabular}{|c|c|c|}
\hline Protocol step & Duration & Flow rate $\mathrm{H}_{2} \mathrm{O}_{2}$ \\
\hline Drying & $3 \mathrm{~min} 28 \mathrm{sec}$ & \\
\hline Gassing I & $8 \mathrm{~min}$ & \\
\hline Dosage I & & $5.0 \mathrm{~g} / \mathrm{min}$ \\
\hline Gassing II & $40 \mathrm{~min}$ & \\
\hline Dosage II & & $3.0 \mathrm{~g} / \mathrm{min}$ \\
\hline
\end{tabular}

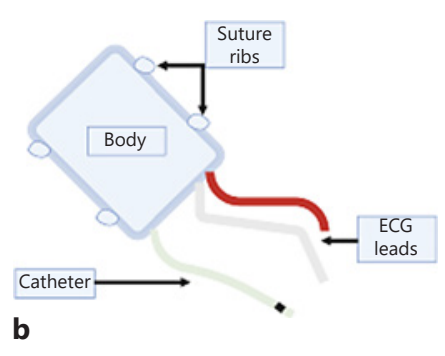

a

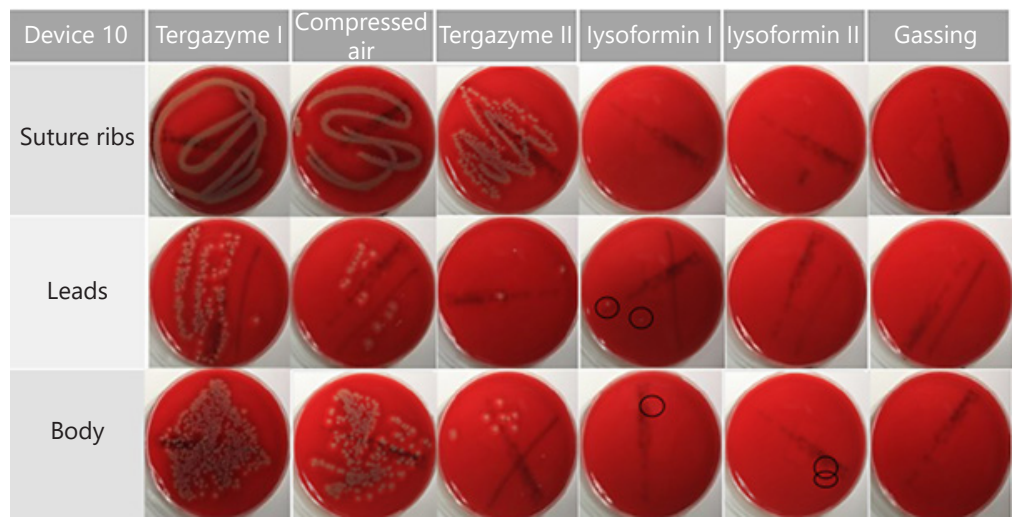

C

\begin{tabular}{|c|c|c|c|}
\hline Device & lysoformin I & lysoformin II & Gassing \\
\hline 1 & 0 & 0 & 0 \\
\hline 2 & 2 & 0 & 0 \\
\hline 3 & 0 & 0 & 0 \\
\hline 4 & 0 & 1 & 0 \\
\hline 5 & 0 & 8 & 0 \\
\hline 6 & 0 & 0 & 0 \\
\hline 7 & 1 & 0 & 0 \\
\hline 8 & 0 & 0 & 0 \\
\hline 9 & 2 & 0 & 0 \\
\hline 10 & 3 & 2 & 0 \\
\hline
\end{tabular}

d

Fig. 2. a Modified protocol for gas sterilisation of an animal-housing isolator with a consumption of $160.0 \mathrm{~g}$ hydrogen peroxide per sterilisation. b Simplified picture of a transponder device and its main components: device body, suture rib, leads, and catheter. c Example of a transponder, No. 10 showing smears at 3 different

There are various technologies for disinfecting and sterilising objects $[9,10]$. In this case, heat, or a combination of heat and steam, is not suitable because of the sensitivity of the technology inside the device. Moreover, the sterilisation protocols of manufacturers are based on the use of ethylene oxide, enzymatic solvents, and cold-sterilising agents. WE used a combination of cold-disinfecting agents and vaporised hydrogen peroxide $\left(\mathrm{H}_{2} \mathrm{O}_{2}\right)$ gas because this is often used for room sterilisation in laboratory animal facilities and is thus often available in animalhousing facilities [6].

\section{Design}

On this basis, we established an in-house protocol for the sterilisation of telemetric devices with hydrogen peroxide gas for further implantations. The technical procedure of our disinfection and sterilisation cycle is shown in Figure 1. In brief, the re-sterilisation process consists of 3 steps: (1) cleaning, (2) disinfection, and (3) sterilisation of the transponder.

Re-Sterilisation of Telemetric Devices locations (suture ribs, body, and leads) at different points in the re-sterilisation process; black circles show the colony-forming units of S. aureus. d Sum of the colony-forming units of a transponder after being soaked twice in $2 \%$ Lysoformin (lysoformin I and II) as well as after successful gas sterilisation (steaming).

The telemetric devices used in this project were implanted for a total time span of 21 days, as part of 2 long-term animal experiments, (i) in pigs (granted by the State Agency for Nature, Environment and Consumer Protection North Rhine-Westphalia [LANUV], Recklinghausen, Germany, protocol No. 81-02.04.2018. A051; EasyTEL+ L-EPTA, EMKA, Paris, France), and (ii) in rats (LANUV, protocol No. 84-02.04.2017.A304, HD-S-11; Data Science International [DSI], New Brighton, MN, USA).

During the cleaning procedure, after explantation from the animal (Fig. 1, step 1), the transponder was transferred to a flask filled with saline solution to macerate any remaining tissue residue on the device. Residue was carefully removed manually after $15 \mathrm{~min}$. The transponder was placed in a new flask filled with $1 \%$ Tergazyme $^{\circledR}$ detergent powder (cat. No. 1304-1, Lot MKCM5800, Alconox Critical Cleaning Experts, New York, NY, USA) for 30 min. Tergazyme is a combination of Alconox and proteases that can be used to remove protein deposits. Afterwards, all surfaces of the transponder were cleaned with a fine brush (especially the suture ribs and leads; Fig. 2b) to remove the biofilm formed during the implantation period. This can be followed by dry storage (at room temperature) until the sterilisation process begins.

During the disinfection steps (Fig. 1, step 2), the device was placed in a sterile dish filled with Tergazyme and placed on a shaking plate (microplate shaker, PMS-1000i, Grant-Bio, Grant Instru- 
ments Ltd., Shepreth, UK) for $30 \mathrm{~min}$. The surface was carefully brushed again, and the transponder was rinsed with sterile distilled water. All surfaces (especially the suture ribs) were spray-cleaned with compressed air (max. 5 bar). This step was repeated using sterile materials. After the second rinsing step, the device was placed in a sterile container, placed on the shaking plate, and covered with 2\% Lysoformin ${ }^{\circledR} 3000$ (Dr. Hans Rosemann GmbH, Berlin, Germany). Lysoformin is a disinfectant and cleaning concentrate for the preparation of wiping and insertion solutions for medical devices and instruments. The transponder was left in $2 \%$ Lysoformin for $30 \mathrm{~min}$. This disinfection step was followed by rinsing with sterile distilled water and repeated.

After the last rinsing step, the sterilisation process was started (Fig. 1, step 3). The transponder was attached to a metal grid, ensuring that the transponder was completely free-swinging and all surfaces were accessible since this is very important for an all-encompassing sterility of the device surface. The grid-device connection was then placed inside a gas sterilisation unit. We used an animalhousing isolator (animal husbandry isolator, THF 3317, unit No. 3688, Ehret GmbH \& Co. KG, Emmendingen, Germany) for sterilisation. The isolator was sealed gas-tight, the gas hoses of the gas generator (model No. 60409, PEA Pharma- u. Elektrotechnik- Anlagenbau $\mathrm{GmbH}$, Koblenz, Germany) were connected to the isolator, and the corresponding gas sterilisation programme was activated. We used $30 \%$ hydrogen peroxide (Perhydrol ${ }^{\circledR}$ for analysis; EMSURE ${ }^{\circledR}$ ISO, Merck KGaA, Darmstadt, Germany) for gas sterilisation.

In general, the sterilisation process consisted of 3 steps. First, the drying step reduced the humidity in the isolator by integrated cooling units. Thus, the dried air enabled more hydrogen peroxide absorption and better gas distribution in the chamber. After this, the gas sterilisation was conducted as 2 sub-steps; in step I (for 8 min), the concentration of hydrogen peroxide was increased quickly to $1,500 \mathrm{ppm}$, and in step II, this concentration was maintained the entire time (40 min; steady state). In the last step of degassing and neutralisation, the concentration was reduced via an integrated catalyst. The catalyst accelerates the decomposition into non-hazardous oxygen and water.

For the re-sterilisation of the transponders, the adaptation of the gas sterilisation step to the sterilisation unit is decisive. As we were using an isolator as a gas sterilisation unit, we adapted a validated protocol for gas sterilisation of this animal-housing isolator (Fig. 2a).

After the programme was successfully completed, the transponder was removed using sterile gloves, and degassed in a biosafety cabinet class II, running for $24 \mathrm{~h}$. This prevented late tissue reactions due to residual hydrogen peroxide gas.

Double packaging is recommended for sterile storage and usage for surgical procedures.

To verify and evaluate the individual steps of the re-sterilisation process, the transponders were inoculated with Staphylococcus aureus before the start of the process. Smear samples were taken during the process at different locations on the device (Fig. 2b-d), and then incubated on tryptic soya agar with $5 \%$ sheep blood (article No. OXPB5012A, Oxoid, Germany). For protocol implementation, 2 additional devices were used. To validate the correct gas sterilisation process, a bio-indicator strip (spore strips with Geobacillus stearothermophilus; article No. 7953; gke-GmbH, Waldems, Germany) was added in the isolator during each process. No growth of the bacterial cultures could be detected after successful completion of the gas sterilisation procedure.

\section{Conclusion}

Our investigations demonstrate that despite 2 Lysoformin treatments, sufficient bacterial disinfection was not achieved. In 10/10 cases, sterility was only achieved by gas sterilisation with hydrogen peroxide.

To investigate functionality and sterility, we re-implanted 4 of the re-sterilised transponders into the pigs for another 21 days, and observed neither undesired tissue reactions along the transponder nor impairment of the measured values compared to the first implantation and after necropsy at the end of the experiments.

Following our protocol, unique re-implantation of reprocessed single-use transponders can be performed without impairing the measurement function, as long as an appropriate battery life can be guaranteed for the scheduled duration of the transponders' use in the animal.

\section{Acknowledgement}

Our thanks to Tanja Pfeffer and Britta Bungardt for their skillful technical assistance.

\section{Statement of Ethics}

The underlying animal experiments of this manuscript were approved by the State Agency for Nature, Environment and Consumer Protection North Rhine-Westphalia (LANUV), Recklinghausen, Germany, with regard to their ethical justification (pigs: protocol No.: 81-02.04.2018.A051; rats: protocol No.: 8402.04.2017.A304).

\section{Conflict of Interest Statement}

The authors have no conflicts of interest to declare.

\section{Funding Sources}

This study was funded by the Deutsche Forschungsgemeinschaft (TO 542/5-1 and TO 542/5-2).

\section{Author Contributions}

L.Z. wrote the protocol and performed the surgeries, re-sterilisation, and protocol evaluation, and also created the images. A.M.K. performed the re-sterilisation and protocol evaluation. L.E. performed the surgeries, gave conceptual input about protocol establishment, created the images, and proofread the paper. R.H.T. initiated the study, got the funding, gave conceptual input of protocol establishment and methods, and proofread the paper. 


\section{References}

1 Butz GM, Davisson RL. Long-term telemetric measurement of cardiovascular parameters in awake mice: a physiological genomics tool. Physiol Genomics. 2001 Mar;5(2):89-97.

2 Wang B, Sun G, Qiao W, Liu Y, Qiao J, Ye W, et al. Long-term blood glucose monitoring with implanted telemetry device in conscious and stress-free cynomolgus monkeys. J Endocrinol Invest. 2017 Sep;40(9):967-77.

3 Liu WJ, Ernst L, Doorschodt B, Bednarsch J, Becker F, Nakatake R, et al. Orthotopic Kidney Auto-Transplantation in a Porcine Model Using 24 Hours Organ Preservation And Continuous Telemetry. J Vis Exp. 2020 Aug;(162).
4 Ruppert S, Vormberge T, Igl BW, Hoffmann M. ECG telemetry in conscious guinea pigs. J Pharmacol Toxicol Methods. 2016 SepOct;81:88-98.

5 Thireau J, Zhang BL, Poisson D, Babuty D. Heart rate variability in mice: a theoretical and practical guide. Exp Physiol. 2008 Jan;93(1):83-94.

6 Benga L, Benten WP, Engelhardt E, Gougoula C, Schulze-Röbbecke R, Sager M. Survival of bacteria of laboratory animal origin on cage bedding and inactivation by hydrogen peroxide vapour. Lab Anim. 2017 Aug;51(4):41221.
7 Costerton JW, Stewart PS, Greenberg EP. Bacterial biofilms: a common cause of persistent infections. Science.1999 May;284(5418):131822.

8 Lebeaux D, Ghigo JM, Beloin C. Biofilm-related infections: bridging the gap between clinical management and fundamental aspects of recalcitrance toward antibiotics. $\mathrm{Mi}$ crobiol Mol Biol Rev. 2014 Sep;78(3):510-43.

9 McEvoy B, Rowan NJ. Terminal sterilization of medical devices using vaporized hydrogen peroxide: a review of current methods and emerging opportunities. J Appl Microbiol. 2019 Nov;127(5):1403-20.

10 Schneider PM. New technologies and trends in sterilization and disinfection. Am J Infect Control. 2013 May;41(5 Suppl):S81-6. 